\title{
The Study of Causes of Chronic Renal Failure in Hemodialysis Patients of Hamadan Hemodialysis Centers in 2018
}

\author{
Mehdi Molavi Vardanjani (iD), ${ }^{1,}$, Danial Shadi ${ }^{2}$, Erfan Gomar $^{2}$ and Narges Kalvandi ${ }^{2,{ }^{* *}}$ \\ ${ }^{1}$ Research Center for Chronic Diseases (Home Care), Faculty of Nursing and Midwifery, Hamadan University of Medical Sciences, Hamadan, Iran \\ ${ }^{2}$ Student Research Committee, Faculty of Nursing and Midwifery, Hamadan University of Medical Sciences, Hamadan, Iran \\ "Corresponding author: Research Center for Chronic Diseases (Home Care), Faculty of Nursing and Midwifery, Hamadan University of Medical Sciences, Hamadan, Iran. Email: \\ m.molavi@umsha.ac.ir \\ "Corresponding author: Student Research Committee, Faculty of Nursing and Midwifery, Hamadan University of Medical Sciences, Hamadan, Iran. Email: \\ kalvandinarges@gmail.com
}

Received 2019 December 31; Accepted 2020 March 06.

\begin{abstract}
Objective: The present study was a retrospective cross-sectional analytical study.

Methods: This study was conducted on 301 hemodialysis patients referred to Hemodialysis centers in Hamadan in 2018 . The tools for collecting information in this research are questions about demographic information (gender, age, educational level, occupational and housing status, marital status of the disease), and a checklist for the cause of chronic renal failure based on high blood pressure, diabetes, kidney disease, and other reasons. Chi-square, Fisher's exact test, and ANOVA and SPSS version 24 software were used to analyze the data in the inferential statistics section.

Results: The results of the study showed that blood pressure, other causes (trauma, drugs, lumbar surgery, etc.), kidney disease, and diabetes were the main causes of chronic renal failure in hemodialysis patients. Prioritization of the causes of chronic renal failure was due to other causes, unspecified causes (15.3\%), history of surgery (6.3\%), anemia (5\%), trauma (4.3\%), shock (7.7\% $2 \%$ ), viral diseases (2\%), drug use and congenital causes (1.3\%), and poisoning of pregnancy and lupus (0.3\%) were among the most important reasons for end-stage renal disease (ESRD) in these patients; also subset renal diseases were the most important causes of chronic renal failure in hemodialysis patients, kidney stones (3.3\%), polycystic kidney (0.7\%) and glomerulonephritis (0.3\%), respectively. Conclusions: According to the results of this study, it can be concluded that by promoting screening methods in patients at risk, prevention of end-stage renal failure can be prevented.
\end{abstract}

Keywords: Chronic Renal Failure, Patient, Hemodialysis

\section{Background}

Chronic renal failure refers to the irreversible impairment of glomerular function permanently (1). Chronic renal failure is one of the chronic diseases that has been on the rise in recent years. According to the latest figures, the incidence of the disease has been 260 cases per million people a year, with an increase of eight percent each year, with the increasing incidence 10 -fold over the past 20 years in the United States. According to statistics from the Ministry of Health and Medical Education, there are currently about 39,000 patients with chronic renal failure in Iran, and the prevalence of chronic renal failure in Iran is reported to be 250 per million $(2,3)$.

It seems that despite many efforts to prevent end-stage renal disease (ESRD), the lack of proper medical and diagnostic facilities and timely referral of patients to physicians and medical centers annually reaches 16,000 pa- tients. Hemodialysis has been replaced as the most common treatment and costs very high in the Iranian health system, so that the cost of treatment per patient in our country is 11053 US \$ (2). In addition, despite the high cost of treatment for this group of patients, they experience high rates of family problems, depression, stress, and death (4). At the same time, they have been able to identify the causes of chronic renal failure and early treatment in advanced countries. They minimize the treatable causes and delay the onset of dialysis (5). Therefore, recognition of these patients in the early stages and special care is considered to be effective in delaying advanced renal failure and decreasing mortality and costs and enhancing the quality of life (6).

In different countries, the causes and risk factors for kidney failure vary according to geographical area, lifestyle, family context, renal failure, gender, race, and 
health facilities available for the diagnosis and treatment of kidney disease $(3,7,8)$. According to the study of Raisifar in Abadan, the most important cause of chronic renal failure was hypertension and diabetes (9). Chronic gastrolonephritis was chronic (10). Therefore, considering the importance of prevention of chronic renal failure, as well as the impact of geographical differences and the role of genetic factors and environmental factors in its development, investigating its underlying factors can determine the prevalence of each of these factors. To help the health system better control this phenomenon (9-11).

Knowing the etiology of chronic renal failure in each region promptly diagnosed and treated the underlying diseases in a timely manner, minimizing its treatable causes and minimizing the onset of dialysis $(12,13)$; It is possible to reduce community health costs and extend the life span of these patients (14). Given the differences in the underlying causes of chronic renal failure in different areas as well as the clinical experience of the researcher in dealing with a large number of patients undergoing permanent hemodialysis as well as the lack of study in this area in Hamadan, the researcher decided to do so.

\section{Objectives}

The aim of this study was to determine the causes of chronic renal failure in patients undergoing hemodialysis in Hamadan in 2018.

\section{Methods}

This retrospective cross-sectional study was performed with 300 hemodialysis patients after the approval of Hamadan University of Medical Sciences ViceChancellor for Research and receiving the code of ethics IR.UMSHA.REC.1397.210. The study population consisted of all chronic renal failure patients undergoing hemodialysis. Patients with chronic renal failure undergoing hemodialysis were referred to Hamadan Hemodialysis centers after obtaining informed written consent and explaining the purpose of the study. Data collection tools included demographic information (sex, age, education, occupational status and residence, marital status, history of illness) and chronic kidney failure etiology checklist based on hypertension, diabetes, kidney disease, and other reasons. The content validity of the questionnaire was used to determine the validity of the questionnaire. By using reference books, the content of the questions was determined, then ten faculty members of Nursing and Midwifery Faculty were asked to comment.
After considering these questions, expert and followup recommendations have been categorized into four subtypes of hypertension, diabetes, history of kidney disease, and other causes, following recommended improvements. Glomerulonephritis, polycystic kidney, and kidney stone were classified as subgroups of kidney disease and shock, anemia, trauma, pregnancy poisoning, viral diseases, major surgical history, congenital causes, drug use, lupus, and other unknowns. Content validity ratio $(\mathrm{cvr}=75 \%)$ and content validity index ( $\mathrm{cvi}=90 \%$ ) were calculated and corroborated by the reliability of the tool. Moreover, the Cronbach's alpha coefficients were related to chronic failure factors to assess the internal consistency of the Cronbach's alpha coefficients of the instruments. All were estimated at 0.85 . The inclusion criteria consisted of at least patients who had a hemodialysis treatment session, native-born patients of the city, availability of medical records, and being the patient in the ESRD stage. The exclusion criteria were the patients who had acute renal failure, or they temporarily underwent hemodialysis without fistula.

Initially, the quota sampling was performed as 150 records from Shahid Beheshti Hospital and 150 returnees from Bessat Hospital as the sample were then randomly selected from among the patients' records archive from 2014 to 2018. The diagnosis of glomerulonephritis may be based on kidney biopsy or a history of proteinuria with hematuria and hypertension. In this study, patients with a history of hypertension $(140 / 90<$ hypertension) long before chronic renal failure were considered to be hypertensive.

In this study, descriptive and inferential statistics were used for data analysis. The collected data were finally entered into SPSS version 24 software and used for all analyzes and statistical modeling. The descriptive statistics section uses a frequency table to describe the categorized data and the central and dispersion criteria, including mean and standard deviation. Chi-square, Fisher exact test, and ANOVA were used to analyze the data. The significance level was set at 0.05 .

\section{Results}

In this study, the information of 301 hemodialysis patients with chronic renal failure was evaluated. The information was collected through the medical records of patients. The results showed that $36.2 \%$ of patients were female, and $63.8 \%$ were male. In addition, $73.8 \%$ of urban and $26.2 \%$ of rural patients had a mean age of $59.08 \%$. The results also showed hypertension (24.9\%), diabetes (5.3\%), and other causes (trauma (4.3\%), medication (1.3\%), congenital causes (1.3\%.) were the other causes of chronic renal failure in hemodialysis patients. Non-renal causes of renal failure were included uncertain (15.3\%), surgical history 
(6.3\%), anemia (5\%), trauma (4.3\%), shock (7.7\%), viral diseases ( $2 \%)$, drug use and congenital causes (1.3\%), and pregnancy and lupus poisoning (0.3\%) were the most important other causes of ESRD in these patients, respectively.

Also, data analysis showed that men who had chronic kidney failure due to hypertension or diabetes had a higher incidence of women. Chi-square test was used to compare the frequency of males and females in each group, which did not show a statistically significant difference $(P=0.176)$ (Table 1$)$.

\section{Discussion}

In the present study, hypertension, other causes, kidney disease, and diabetes were the causes of chronic renal failure in hemodialysis patients.

However, in some studies, the most well-known causes of renal failure in patients under study were blood pressure and diabetes, respectively. In the Rezaiean Langroudi and Oshvandi study (13), the most common causes of idiopathic renal failure were diabetes and hypertension. In another study (14) performed on Guilan hemodialysis patients, blood pressure, glomerulonephritis, urinary stone, and polycystic kidney were $8.7 \%, 10.9 \%$, and $26.1 \%$, respectively. Another study (15) also reported that the most common causes of chronic kidney failure were hemodialysis patients with blood pressure and diabetes. In contrast, a US study found the most common causes of the disease to be $50 \%$ diabetes (1). In a study by Zhang et al. (16) in China, the most common causes of ESRD abnormalities in hemodialysis patients were glomerulonephritis, hypertension, and diabetes, respectively.

Also, in a study of 800 patients with chronic kidney failure in Ivory Coast (17), chronic glomerulonephritis (49.1\%) and hypertension (4.25\%) were the most important causes of chronic kidney disease. A study among patients of Western countries (17) reported that the causes of the disease were $5.22 \%$ diabetes, $1.1 \%$ hypertension, $6.10 \%$ diabetes, and hypertension, and $6.27 \%$ with unknown cause. The results of a study among hemodialysis patients in Mashhad (15) also show that the most common cause of the disease is hypertension, with $32.2 \%$ and diabetes with $22.3 \%$. What can be deduced from these reports is that various factors such as location, race, diet, etc. can contribute to this problem that need to be studied in particular.

In this study, several hypotheses can be raised about the high blood pressure in hemodialysis patients in this study: first, the number of diabetic patients with endstage nephropathy in this area is lower, which may indicate appropriate attention. It is more likely to have diabetes in this region or, conversely, higher mortality of diabetes nephropathy, and as a result, is less observed in our study population. The second hypothesis is that there is insufficient attention to blood donation in this area, and issues such as the type of diet, products, and food products of this region can cause a high incidence of blood donation in the area, which needs further studies. Research findings in response to the overall goal indicate that the etiologic factors affecting renal failure in hemodialysis patients are variables such as occupation, sex, age, and place of residence. The results showed that $63.8 \%$ of patients were male.

In a study by Khader et al. (12) among patients in Western countries, the ratio of male to female patients was 7.57\% versus $4.42 \%$, and in the study of Biavo et al. (18), 362 was female, and 642 was male. In the USRD (United States Rena Data System) statistics, the ratio of male to female is also 1/2 to 1(18). In the present study, hypertension was the most important cause of chronic renal failure in both male and female gender groups. Hypertension appears to be due to a lack of awareness and function of these individuals to exacerbate its long-term effects, so controlling hypertension and lifestyle changes is important in other countries. Furthermore, its effects have been delayed, thus emphasizing the use of mass education at the grassroots level and the motivation to change their behavior and lifestyle and their easy access to health care facilities.

However, in the study of Ghorbani et al. (19), the main causes of diabetes in men were $6.28 \%$ and hypertension $5.26 \%$ and in women, hypertension $4.36 \%$ and diabetes $27.2 \%$, respectively. In the study of. Monfared et al., The most common causes of chronic kidney failure in men were hypertension of 3.202 and glomerulonephritis of 4.152, while in women, hypertension was 7.312 and diabetes was 9.102 of the main causes (17). According to the findings of the Kher (14) study, the mean age of dialysis patients was 59.08. The average age in most developed countries is $60-63$ years. The results show that the average age of diagnosis in the city is slightly higher than in other parts of the country and slightly lower than in developed countries. However, this average difference was not statistically significant. This is despite the fact that, according to the Dialysis Society of Japan, the average age of dialysis patients was 63.3 years, which may also be due to the higher quality of health care in Japan. Patients' age is also a point to note, as renal failure, a chronic disease, affects older people with specific life and mental conditions.

In the present study, there was a significant relationship between occupational status and chronic renal failure in hemodialysis patients, with $33 \%$ of hemodialysis patients being unemployed and $26.9 \%$ having selfemployment, which could be justified. She said these patients are unable to work in day-to-day office positions due to the number of hemodialysis therapies and burnout, and 
Table 1. The Relationship Between Gender and Causes of Chronic Kidney Failure

\begin{tabular}{|c|c|c|c|c|c|c|}
\hline & \multicolumn{6}{|c|}{ Cause of Renal Failure } \\
\hline & Hypertension & Diabetes & Renal Disease & other Causes & $\chi^{2}$ Statistics & PValue \\
\hline Sex & & & & & 94.4 & 176.0 \\
\hline Male & 62 & 31 & 42 & 57 & & \\
\hline Female & 31 & 29 & 19 & 30 & & \\
\hline
\end{tabular}

prolonged exposure to the disease and dialysis process, and usually turn to freelance work and eventually become unemployed. As Globan declares, long-term dialysis creates medical, social, and health problems. It is an emotion that leads to a feeling of dependence and loss of a job. According to the findings of the study, the most frequent cases of people with chronic kidney failure were those living in urban areas (73.8\%). However, the difference between the frequency of people with chronic renal failure based on the causes of failure in urban and rural areas was not statistically significant.

\subsection{Conclusions}

Finally, it is recommended to prevent people with endstage renal disease due to the increasing prevalence of CKD, by improving screening methods, and in particular, the faster referral of patients at risk. Completing statistical information within the Ministry of Health's National Plans for Kidney Disease Monitoring is essential. One of the important things about educating people through social media is to get acquainted with the symptoms of diabetes and hypertension and specifically to refer patients regularly to a doctor.

\section{Acknowledgments}

The authors of this article sincerely thank the Deputy of Research and Technology of Hamadan University of Medical Sciences and staff of Bessat and Shahid Beheshti hospitals, especially the staff of the dialysis units and all of the patients who helped us in this study.

\section{Footnotes}

Authors' Contribution: Narges Kalvandi and Danial Shadi collected data. Zahra Maghsoodi and Mehdi Molavi Vardanjani wrote the article. All authors read and approved the final manuscript.

Conflict of Interests: There is no conflict of interest.

Ethical Approval: This study was approved by Hamadan University of Medical Sciences ViceChancellor for Research and received the code of ethics IR.UMSHA.REC.1397.210.
Funding/Support: Vice-Chancellor for Research of Hamadan University of Medical Sciences supported this study.

Informed Consent: Patients with chronic renal failure undergoing hemodialysis were referred to Hamadan Hemodialysis Centers after obtaining informed written consent and explaining the purpose of the study.

\section{References}

1. Lederer E, Ouseph R. Chronic kidney disease. Am J Kidney Dis 2007;49(1):162-71. doi:10.1053/j.ajkd.2006.09.021. [PubMed:17185158].

2. Mortazavi F, Rafiee A. Etiology of pediatric chronic kidney diseases in north-west of Iran. Pak J Biol Sci. 2010;13(9):456-9. doi: 10.3923/pjbs.2010.456.459. [PubMed: 20973401].

3. Maw TT, Fried L. Chronic kidney disease in the elderly. Clin Geriatr Med. 2013;29(3):611-24. doi: 10.1016/j.cger.2013.05.003. [PubMed: 23849011].

4. Coresh J, Selvin E, Stevens LA, Manzi J, Kusek JW, Eggers P, et al. Prevalence of chronic kidney disease in the United States. JAMA. 2007;298(17):2038-47. doi: 10.1001/jama.298.17.2038. [PubMed: 17986697].

5. White W, Cove-Smith A. Kidney disease in the elderly. Medicine. 2015;43(8):489-92. doi:10.1016/j.mpmed.2015.05.002.

6. Abdel-Kader K, Palevsky PM. Acute kidney injury in the elderly. Clin Geriatr Med. 2009;25(3):331-58. doi: 10.1016/j.cger.2009.04.001. [PubMed: 19765485]. [PubMed Central: PMC2748997].

7. Bello AK, Nwankwo E, El Nahas AM. Prevention of chronic kidney disease: A global challenge. Kidney Int Suppl. 2005;(98):S11-7. doi: 10.1111/j.1523-1755.2005.09802.x. [PubMed:16108964].

8. Dowling TC, Wang ES, Ferrucci L, Sorkin JD. Glomerular filtration rate equations overestimate creatinine clearance in older individuals enrolled in the Baltimore Longitudinal study on aging: Impact on renal drug dosing. Pharmacotherapy. 2013;33(9):91221. doi: 10.1002/phar.1282. [PubMed: 23625813]. [PubMed Central: PMC3732548].

9. Tohidi M, Hasheminia M, Mohebi R, Khalili D, Hosseinpanah F, Yazdani B, et al. Incidence of chronic kidney disease and its risk factors, results of over 10 year follow up in an Iranian cohort. PLoS One. 2012;7(9). e45304. doi: 10.1371/journal.pone.0045304. [PubMed: 23028919]. [PubMed Central: PMC3459968].

10. Porter CJ, Moppett IK, Juurlink I, Nightingale J, Moran CG, Devonald MA. Acute and chronic kidney disease in elderly patients with hip fracture: prevalence, risk factors and outcome with development and validation of a risk prediction model for acute kidney injury. BMC Nephrol. 2017;18(1):20. doi: 10.1186/s12882-017-0437-5. [PubMed: 28088181]. [PubMed Central: PMC5237525].

11. Mehrabi S, Sarikhani S, Roozbeh J. Sleep quality in patients undergoing long-term hemodialysis using the pittsburgh sleep quality index. Nephro Urolo Mon. 2017;9(2). e44278. doi: 10.5812/numonthly.44278.

12. Khader MI, Snouber S, Alkhatib A, Nazzal Z, Dudin A. Prevalence of patients with end-stage renal disease on dialysis in the West Bank, 
Palestine. Saudi J Kidney Dis Transpl. 2013;24(4):832-7. doi: 10.4103/13192442.113913. [PubMed: 23816745].

13. Rezaiean Langroudi R, Oshvandi K. Risk factors for chronic renal failure in hemodialysis patients referring to hospitals affiliated to Hamadan University of Medical Sciences. Aflak Res Papers. 2008;7(12).

14. Kher V. End-stage renal disease in developing countries. Kidney Int 2002;62(1):350-62. doi: 10.1046/j.1523-1755.2002.00426.x. [PubMed: 12081600].

15. Cano Romer A, Morlans M, López Plana A, Llosa Dessy L, López Expósito $\mathrm{F}$, Espona Barris R, et al. Prevalencia de insuficiencia renal crónica en atención primaria. Atención Primaria. 2002;29(2):90-6. doi: 10.1016/s0212-6567(02)70512-x.

16. Zhang QL, Rothenbacher D. Prevalence of chronic kidney disease in population-based studies: Systematic review. BMC Public Health. 2008;8:117. doi: 10.1186/1471-2458-8-117. [PubMed: 18405348]. [PubMed
Central: PMC2377260].

17. Yao HK, Konan SD, Sanogo S, Diopoh SP, Diallo AD. Prevalence and risk factors of chronic kidney disease in Cote D'Ivoire: An analytic study conducted in the department of internal medicine. Saudi J Kidney Dis Transpl. 2018;29(1):153-9. doi: 10.4103/1319-2442.225201. [PubMed: 29456222].

18. Biavo BM, Cunha LM, Araujo ML, Ribeiro MM, Sachs A, Uezima CB, et al. Nutritional and epidemiological aspects of patients with chronic renal failure undergoing hemodialysis from Brazil, 2010. J Bras Nefrol. 2012;34(3):206-15. doi: 10.5935/0101-2800.20120001. [PubMed: 23099825].

19. Ghorbani Z, Sharifi S. The frequency of underlying conditions for chronic renal failure in patients undergoing dialysis in shohada hospital in bushehr. Nurs Vulnerables. 2015;2(4):46-54. 